\title{
Locally Anisotropic Supergravity and Gauge Gravity on Noncommutative Spaces
}

\author{
S. I. Vacaru *, I. A. Chiosa *, and Nadejda A. Vicol ${ }^{\diamond}$ \\ * Institute of Applied Physics, Academy of Sciences, \\ Academy str. 5, Chişinău MD2028, Republic of Moldova \\ $\diamond$ Faculty of Mathematics and Informatics, gr. $33 \mathrm{MI}$, \\ State University of Moldova, Mateevici str. 60, Chişinău MD2009
}

November 24, 2000

\begin{abstract}
We outline the the geometry of locally anisotropic (la) superspaces and la-supergravity. The approach is backgrounded on the method of anholonomic superframes with associated nonlinear connection structure. Following the formalism of enveloping algebras and star product calculus we propose a model of gauge la-gravity on noncommutative spaces. The corresponding Seiberg-Witten maps are established which allow the definition of dynamics for a finite number of gravitational gauge field components on noncommutative spaces.
\end{abstract}

\section{Introduction}

Locally anisotropic supergravity was developed as a model of supergravity with anholonomic superframes and associated nonlinear connection (N-connection) structure [13]. This model contain as particular cases supersymmetric KaluzaKlein and generalized Lagrange and/or Finsler gravities and for nontrivial curvatures the $\mathrm{N}$-connection describes splittings from higher to lower dimensions of (super) spaces and generic anholonomic local anisotropies.

In order to avoid the problem of formulation of gauge theories on noncommutative spaces [3, 10, 5, 7] with Lie algebra valued infinitesimal transformations and with Lie algebra valued gauge fields the authors of [6] suggested to use enveloping algebras of the Lie algebras for setting this type of gauge theories and showed that in spite of the fact that such enveloping algebras are

\footnotetext{
*e-mail: svacaru@phys.asm.md, vacaru@lises.asm.md, sergiu_vacaru@yahoo.com
} 
infinite-dimensional one can restrict them in a way that it would be a dependence on the Lie algebra valued parameters and the Lie algebra valued gauge fields and their spacetime derivatives only.

A still presented drawback of noncommutative geometry and physics is that there is not yet formulated a generally accepted approach to interactions of elementary particles coupled to gravity. There are improved Connes-Lott and Chamsedine-Connes models of nocommutative geometry [2] which yielded action functionals typing together the gravitational and Yang-Mills interactions and gauge bosons the Higgs sector (see also the approaches [4] and [8]).

In this paper we outline the geometry of locally anisotropoc supergravity and follow the method of restricted enveloping algebras [5, 6] and construct gauge gravitational theories by stating corresponding structures with semisimple or nonsemisimple Lie algebras and their extensions. We consider power series of generators for the affine and non linear realized de Sitter gauge groups and compute the coefficient functions of all the higher powers of the generators of the gauge group which are functions of the coefficients of the first power. Such constructions are based on the Seiberg-Witten map [10] and on the formalism of $*$-product formulation of the algebra 18 when for functional objects, being functions of commuting variables, there are associated some algebraic noncommutative properties encoded in the $*$-product. The concept of gauge gravity theory on noncommutative spaces is introduced in a geometric manner [0] by defining the covariant coordinates without speaking about derivatives and this formalism was developed for quantum planes [17. We prove the existence for noncommutative spaces of gauge models of gravity which agrees with usual gauge gravity theories [14 being equivalent, or extending, the general relativity theory (see works [9, 11] for locally isotropic spaces and corresponding reformulations and generalizations respectively for anholonomic frames [15] and locally anisotropic (super) spaces [16]) in the limit of commuting spaces.

\section{Locally Anisotropic Supergravity}

Let us consider a vector superbundle (vs-bundle) $\widetilde{E}$ over a supermanifold (s-manifold) $\widetilde{M}$ with surjective projection $\pi_{E}: \widetilde{E} \rightarrow \widetilde{M}$ (for simplicity, all constructions are locally trivial). The local supersymmetric coordinates (scoordinates) on $\widetilde{E}$ and $\widetilde{M}$ are denoted respectively $u=(x, y)=\left\{u^{\alpha}=\right.$ $\left(x^{I}, y^{A}\right)$, where $x=\left\{x^{I}=\left(x^{i}, x^{\widehat{i}}\right)\right\}$ are (even,odd) coordinates on $\widetilde{M}$ and $y=\left\{y^{A}=\left(y^{a}, y^{\widehat{a}}\right)\right\}$ are (even,odd) coordinates in fibers of $\pi_{E}$ (indices run values defined by even and odd dimensions of corresponding submanifolds). Latin s-indices $I, J, K, L, M, \ldots$ and $A, B, C, D, \ldots$ will be used respectively for base and fiber components.

A nonlinear connection (N-connection) structure which defines a global decomposition of $T \widetilde{E}$ into horizontal, $H \widetilde{E}$, and vertical parts, $V \widetilde{E}$,

$$
N: T \widetilde{E}=H \widetilde{E} \oplus V \widetilde{E}
$$


The coefficients of a $\mathrm{N}$-connection $N_{I}^{A}(u)$ determin the locally adapted s-frame (basis, in brief la-frame)

$$
\delta_{\alpha}=\delta / \delta u^{\alpha}=\left(\delta_{I}=\delta / \delta x^{I}=\partial_{I}-N_{I}^{B}(u) \partial_{B}, \partial_{A}\right),
$$

where $\partial_{I}=\partial / \partial x^{I}, \partial_{A}=\partial / \partial y^{A}$ are partial s-derivatives, and the dual s-frame

$$
\delta^{\alpha}=\delta u^{\alpha}=\left(d^{I}=\delta x^{I}=d x^{I}, \delta^{A}=\delta y^{A}=d y^{A}+N_{I}^{A}(u) d x^{I}\right) .
$$

The s-frame (2) is anholonomic

$$
\left[\delta_{J}, \delta_{K}\right\}=\delta_{J} \delta_{K}-(-)^{|J K|} \delta_{K} \delta_{J}=\Omega_{J K}^{A} \partial_{A},
$$

where $|J K|=|J| \cdot|K|$ is defined by the parity of indices and we write $(-)^{|J K|}$ instead $(-1)^{|J K|}$, with anholonomy coefficients coinciding with the N-connection curvature

$$
\Omega_{J K}^{A}=\delta_{K} N_{J}^{A}-(-)^{|J K|} \delta_{J} N_{K}^{A}
$$

The geometrical objects on $\widetilde{E}$ are given with respect to la-basis (2) and (3) or their tensor products and called ds-tensors, ds-connections (for some additional linear connections), $\mathrm{d}$-spinors and so on. For instance, a metric ds-tensor is written

$$
\widetilde{g}=g_{\alpha \beta} \delta^{\alpha} \otimes \delta^{\beta}=g_{I J} d^{I} \otimes d^{J}+g_{A B} \delta^{A} \otimes \delta^{B} .
$$

The Lagrange and Finsler ds-metrics can be modelled on a locally anisotropic superspace if vs-bundle $\widetilde{E}$ over a s-manifold $\widetilde{M}$ is substituted by the tangent s-bundle $T \widetilde{M}$ and the coefficients of ds-metric (丑) are taken respectively

$$
g_{I J}(u)=\frac{1}{2} \frac{\partial^{2} \mathcal{L}(u)}{\partial y^{I} \partial y^{L}} \text { and } g_{I J}(u)=\frac{1}{2} \frac{\partial^{2} F^{2}(u)}{\partial y^{I} \partial y^{L}}
$$

where the s-Lagrangian $\mathcal{L}: T \widetilde{M} \rightarrow \Lambda$ is a s-differentiable function on $T \widetilde{M}$, and $F$ is a Finsler s-metric function on $T \widetilde{M}$.

A linear distinguished connection $D$, d-connection, in sv-bundle $\widetilde{E}$ is a linear connection which preserves by parallelism the horizontal (h) and vertical (v) distribution (1).

A d-connection $D \Gamma=\left\{\Gamma_{\beta \gamma}^{\alpha}=(L, \widetilde{L}, \widetilde{C}, C)\right\}$, is determined by its invariant hh-, hv-, vh- and vv-components, where

$$
\begin{aligned}
& D_{\left(\delta_{K}\right)} \delta_{J}=L_{J K}^{I}(u) \delta_{I}, D_{\left(\delta_{K}\right)} \partial_{B}=L_{B K}^{A}(u) \partial_{A}, \\
& D_{\left(\partial_{C}\right)} \delta_{J}=C_{J C}^{I}(u) \delta_{I}, D_{\left(\partial_{C}\right)} \partial_{B}=C_{B C}^{A}(u) \partial_{A} .
\end{aligned}
$$

There is a canonical $\mathrm{d}$-connection ${ }^{(c)} \Gamma$ defined by the coefficients of $\mathrm{d}-$ metric (4) and of $\mathrm{N}$-connection and satisfying the metricity condition $D \tilde{g}=0$,

$$
\begin{aligned}
{ }^{(c)} L_{J K}^{I} & =\frac{1}{2} g^{I H}\left(\delta_{K} g_{H J}+\delta_{J} g_{H K}-\delta_{H} g_{J K}\right), \\
{ }^{(c)} L_{B K}^{A} & =\partial_{B} N_{K}^{A}+\frac{1}{2} h^{A C}\left(\delta_{K} H_{B C}-\left(\partial_{B} N_{K}^{D}\right) h_{D C}-\left(\partial_{C} N_{K}^{D}\right) h_{D B}\right), \\
{ }^{(c)} C_{J C}^{I} & =\frac{1}{2} g^{I K} \partial_{C} g_{J K},{ }^{(c)} C_{B C}^{A}=\frac{1}{2} h^{A D}\left(\partial_{C} h_{D B}+\partial_{B} h_{D C}-\partial_{D} h_{B C}\right) .
\end{aligned}
$$


The torsion $T_{\beta \gamma}^{\alpha}$ of a d-connection, $T(X, Y)=[X, D Y\}-[X, Y\}$, where $X$ and $Y$ are ds-vectors and by $[\ldots\}$ we denote the s-anticommutator, is decomposed into hv-invariant ds-torsions

$$
\begin{aligned}
& h T\left(\delta_{K}, \delta_{J}\right)=T_{J K}^{I} \delta_{I}, v T\left(\delta_{K}, \delta_{J}\right)=\widetilde{T}_{J K}^{A} \delta_{I}, h T\left(\partial_{A}, \delta_{J}\right)=\widetilde{P}_{J A}^{I} \delta_{I}, \\
& v T\left(\partial_{B}, \delta_{J}\right)=P_{J B}^{A} \partial_{A}, \quad v T\left(\partial_{C}, \partial_{B}\right)=S_{B C}^{A} \partial_{A},
\end{aligned}
$$

with coefficients

$$
\begin{aligned}
& T_{J K}^{I}=L_{J K}^{I}-(-)^{|J K|} L_{K J}^{I}, \widetilde{T}_{J K}^{A}=\delta_{K} N_{J}^{A}-(-)^{|K J|} \delta_{J} N_{K}^{A}, \\
& \widetilde{P}_{J A}^{I}=C_{J A}^{I}, P_{J B}^{A}=\partial_{B} N_{J}^{A}-L_{B J}^{A}, S_{B C}^{A}=C_{B C}^{A}-(-)^{|B C|} C_{C B}^{A} .
\end{aligned}
$$

The even and odd components of ds-torsions (6) can be specified in explicit form by using decompositions of indices into even and odd parts, $I=(i, \widehat{i}), A=$ $(a, \widehat{a})$ and so on.

The curvature $R_{\beta \gamma \tau}^{\alpha}$ of a d-connection, $R(X, Y) Z=D_{[X} D_{Y\}} Z-D_{[X, Y\}} Z$, where $X, Y, Z$ are ds-vectors, splits into hv-invariant ds-torsions

$$
\begin{aligned}
& R\left(\delta_{K}, \delta_{J}\right) \delta_{H}=R_{H J K}^{I} \delta_{I}, R\left(\delta_{K}, \delta_{J}\right) \partial_{B}=R_{B J K}^{A} \partial_{A}, \\
& R\left(\partial_{C}, \delta_{K}\right) \delta_{J}=\widetilde{P}_{J K C}^{I} \delta_{I}, R\left(\partial_{C}, \delta_{K}\right) \partial_{B}=P_{B K C}^{A} \\
& R\left(\partial_{C}, \partial_{B}\right) \delta_{J}=\widetilde{S}_{J B C}^{I} \delta_{I}, R\left(\partial_{D}, \partial_{C}\right) \partial_{B}=S_{B C D}^{A} \partial_{A}
\end{aligned}
$$

where the coefficients are computed

$$
\begin{aligned}
R_{M J K}^{I} & =\delta_{[K} L_{|M| J\}}^{I}+L_{M J}^{W} L_{W K}^{I}-(-)^{|K J|} L_{M K}^{W} L_{W J}^{I}+C_{K A}^{I} W_{J K}^{A}, \\
\widehat{R}_{B J K}^{A} & =\delta_{[K} L_{|B| J\}}^{A}+L_{B J}^{C} L_{C K}^{A}-(-)^{|K J|} L_{B K}^{C} L_{C J}^{A}+C_{B C}^{A} W_{J K}^{C}, \\
\widetilde{S}_{J B C}^{I} & =\partial_{C} C_{J B}^{I}-(-)^{|B C|} \partial_{B} C_{J C}^{I}+C_{J B}^{H} C_{H C}^{I}-(-)^{|B C|} C_{J C}^{H} C_{H B}^{I}, \\
S_{B C D}^{A} & =\partial_{D} C_{B C}^{A}-(-)^{|C D|} \partial_{C} C_{B D}^{A}+C_{B C}^{E} C_{E D}^{A}-(-)^{|C D|} C_{B D}^{E} C_{E C}^{A}, \\
\widetilde{P}_{J K A}^{I} & =\partial_{A} L_{J K}^{I}-C_{J A \mid K}^{I}+C_{J B}^{I} P_{K A}^{B}, \\
P_{B K C}^{A} & =\partial_{C} L_{B K}^{A}-C_{B C \mid K}^{A}+C_{B D}^{A}{ }_{B C}{ }_{K C}^{D},
\end{aligned}
$$

where, for instance,

$$
\begin{aligned}
\delta_{[K} L_{|M| J\}}^{I} & =\delta_{K} L_{M J}^{I}-(-)^{|K J|} \delta_{J} L_{M K}^{I}, \\
C^{I}{ }_{J A \mid K} & =\delta_{K} C^{I}{ }_{J A}+L_{M K}^{I}{ }_{M A} C_{I A}^{M}-L_{J K}^{M} C_{M A}^{I}-L_{A K}^{B} C_{J B}^{I} .
\end{aligned}
$$

The even and odd components of ds-curvatures are computed by splitting indices into even and odd parts.

The torsion and curvature of a d-connection $D$ on a sv-bundle satisfy the identities

$$
\begin{aligned}
\sum_{S C}\left[\left(D_{X} T\right)(Y, Z)-R(X, Y) Z+T(T(X, Y), Z)\right] & =0, \\
\sum_{S C}\left[\left(D_{X} R\right)(U, Y, Z)-R(T(X, Y), Z) U\right] & =0,
\end{aligned}
$$


where $\sum_{S C}$ means supersymmetric cyclic sums over ds-vectors $X, Y, Z$ and $U$, from which the generalized Bianchi and Ricci identities follow [1-3].

The Ricci ds-tensor $R_{\beta \gamma}=R_{\beta \gamma \alpha}^{\alpha}$ has hv-invariant components

$$
\begin{aligned}
& R_{I J}=R_{I J K}^{K}, R_{I A}=-{ }^{(2)} P_{I A}=-(-)^{|K A|} \widetilde{P}_{I K A}^{K} \\
& R_{A I}={ }^{(1)} P_{A I}=P_{A I B}^{B}, R_{A B}=S_{A B C}^{C}=S_{A B} .
\end{aligned}
$$

If a ds-metric (ब1) is defined on $\widetilde{E}$, we can introduce the supersymmetric scalar curvature

$$
\widehat{R}=g^{\alpha \beta} R_{\alpha \beta}=R+S,
$$

where $R=g^{I J} R_{I J}$ and $S=h^{A B} S_{A B}$.

The simplest model of locally anisotropic supergravity (la-supergravity) was constructed by postulating a variant of supersymmetric Einstein-Cartan theory on LAS-space $\widetilde{E}$, which in invariant hv-components has the fundamental s-field equations

$$
\begin{aligned}
& R_{I J}-\frac{1}{2}(R+S-\lambda) g_{I J}=k_{1} \Upsilon_{I J},{ }^{(1)} P_{A I}=k_{1} \Upsilon_{A I} \\
& S_{A B}-\frac{1}{2}(R+S-\lambda) h_{A B}=k_{1} \Upsilon_{A B},{ }^{(2)} P_{I A}=-k_{1} \Upsilon_{I A},
\end{aligned}
$$

and

$$
T_{\beta \gamma}^{\alpha}+\delta_{\beta}^{\alpha} T_{\gamma \tau}^{\tau}-(-)^{|\beta \gamma|} \delta_{\gamma}^{\alpha} T_{\beta \tau}^{\tau}=k_{2} Q_{\beta \gamma}^{\alpha},
$$

where $\lambda$ is the cosmological constant, $k_{1,2}$ are respective interaction constants $\Upsilon_{\alpha \beta}$ is the energy-momentum ds-tensor and $Q_{\beta \gamma}^{\alpha}$ is defined by the supersymmetric spin-density.

The bulk of theories of locally isotropic s-gravity are formulated as gauge supersymmetric models based on supervielbein formalism. Similar approaches to la-supergravity on vs-bundles can be developed by considering arbitrary s-frames $B_{\underline{\alpha}}(u)=\left(B_{\underline{I}}(u), B_{\underline{C}}(u)\right)$ adapted to the N-connection structure on a vs-bundle $\widetilde{E}=\widetilde{E}^{m, l}$ over s-manifold $\widetilde{M}=\widetilde{M}^{n, k}$ where $(m, l)$ and $(n, k)$ are respective (even, odd) dimensions of s-manifolds. A s-frame $B_{\underline{\alpha}}(u)$ is related with a standard la-frame (2) via transforms $\delta_{\alpha}=A_{\alpha} \underline{\alpha}(u) \underline{B}_{\underline{\alpha}}(u)$, where s-matrices $A_{\alpha}^{\underline{\alpha}}(u)=\left(\begin{array}{cc}A_{I}^{\underline{I}} & 0 \\ 0 & A_{C}^{\underline{C}}\end{array}\right)$ take values into a super Lie group $G L_{n, k}^{m, l}(\Lambda)=G L(n, k, \Lambda) \oplus G L(m, l, \Lambda)$ (on superspaces the graded Grassmann algebra with Euclidean topology, denoted by $\Lambda$, substitutes the real and complex number fields).

We denote by $L N(\widetilde{E})$ the set of all adapted to $\mathrm{N}$-connection s-frames in all points of vs-bundle $\widetilde{E}$ and consider the s-bundle of linear adapted s-frames on $\widetilde{E}$ defined as the principal s-bundle

$$
\mathcal{L} N(\widetilde{E})=\left(L N(\widetilde{E}), \pi_{L}: L N(\widetilde{E}) \rightarrow \widetilde{E}, G L_{n, k}^{m, l}(\Lambda)\right),
$$

for a surjective $\mathrm{s}^{-}$map $\pi_{L}$. The canonical basis of standard distinguished $\mathrm{s}^{-}$ generators $I_{\widehat{\alpha}} \rightarrow I_{\underline{\beta}}^{\underline{\alpha}}=\left(\begin{array}{cc}I \frac{I}{\underline{J}} & 0 \\ 0 & I_{\underline{B}}^{\frac{A}{B}}\end{array}\right)$ for the super Lie algebra $\mathcal{G} L_{n, k}^{m, l}(\Lambda)$ of the 
structural s-group $G L_{n, k}^{m, l}(\Lambda)$ satisfy s-commutation rules $\left[I_{\widehat{\alpha}}, I_{\widehat{\beta}}\right\}=f_{\widehat{\alpha} \widehat{\beta}}{ }^{\widehat{\gamma}} I_{\widehat{\gamma}}$. On $\mathcal{L} N(\widetilde{E})$ we consider the d-connection 1-form

$$
\mathcal{F}=\Gamma \underline{\underline{\beta} \gamma}(u) I_{\underline{\alpha}}^{\underline{\beta}} \delta u^{\gamma},
$$

where

$$
\Gamma_{\underline{\beta} \gamma}^{\underline{\alpha}}(u)=A_{\alpha}^{\underline{\alpha}} A_{\underline{\beta}}^{\beta} \Gamma_{\beta \gamma}^{\alpha}+A_{\beta}^{\underline{\alpha}} \delta_{\gamma} A_{\underline{\beta}}^{\beta},
$$

$\Gamma_{\beta \gamma}^{\alpha}$ are the components of canonical variant of d-connection (5) and the smatrix $A_{\underline{\beta}}^{\beta}$ is inverse to $A_{\alpha}^{\underline{\alpha}}$.

The curvature $\mathcal{B}$ of the $\mathrm{d}$-connection (10)

$$
\mathcal{B}=\delta \mathcal{F}+\mathcal{F} \wedge \mathcal{F}=R_{\underline{\underline{\alpha}} \gamma \tau}^{\underline{\underline{\beta}}} I_{\underline{\beta}} \delta u^{\gamma} \wedge \delta u^{\tau}
$$

has the coefficients $R_{\underline{\alpha} \gamma \tau}^{\underline{\beta}}=A_{\underline{\alpha}}^{\alpha}(u) A_{\beta}^{\underline{\beta}}(u) R_{\alpha \gamma \tau}^{\beta}$, where $R_{\alpha \gamma \tau}^{\beta}$ are defined by ds-curvatures (7).

Aside from $\mathcal{L} N(\widetilde{E})$ with vs-bundle $\widetilde{E}$ is naturally related another sbundle, the bundle of adapted to $\mathrm{N}$-connection affine s-frames

$$
\mathcal{A N}(\widetilde{E})=\left(A N(\widetilde{E}), \pi_{A}: A N(\widetilde{E}) \rightarrow \widetilde{E}, A F_{n, k}^{m, l}(\Lambda)\right),
$$

with the affine strucural s-group $A F_{n, k}^{m, l}(\Lambda)=G L_{n, k}^{m, l}(\Lambda) \odot \Lambda^{n, k} \oplus \Lambda^{m, l}$.

The d-connection $\mathcal{F}(10)$ in $\mathcal{L} N(\widetilde{E})$ induces in a linear Cartan d-connection $\overline{\mathcal{F}}=(\mathcal{F}, \chi)$, in $\mathcal{A} N(\widetilde{E})$, where $\chi=e_{\underline{\alpha}} \otimes A_{\alpha}^{\underline{\alpha}}(u) \delta u^{\alpha}, e_{\underline{\alpha}}$ is the standard basis in $\Lambda^{n, k} \oplus \Lambda^{m, l}$, and, in consequence, the curvature $\mathcal{B}$ (11) in $\mathcal{L} N(\widetilde{E})$ induces the pair (curvature, torsion) $\overline{\mathcal{B}}=(\mathcal{B}, \mathcal{T})$ in $\mathcal{A} N(\widetilde{E})$, where

$$
\mathcal{T}=\delta \chi+[\mathcal{F} \wedge \gamma\}=\mathcal{T} \frac{\alpha}{\beta \gamma} e_{\underline{\alpha}} \delta u^{\beta} \wedge \delta u^{\gamma},
$$

when $\mathcal{T}_{\beta} \frac{\alpha}{\gamma}=A_{\alpha} \underline{\alpha} T_{\beta \gamma}^{\alpha}$ is defined by the coefficients of $\mathrm{d}$-torsions (6) .

By using the ds-metric (雨) in $\widetilde{E}$ one defines the (dual for s-forms) Hodge operator $*_{\tilde{g}}$. Let the operator $*_{\tilde{g}}^{-1}$ be inverse to $*_{\tilde{g}}$ and $\widehat{\delta}_{\tilde{g}}$ be the adjoint to the absolute derivation $\widehat{\delta}$ (associated to the scalar product of ds-forms) specified for $(\mathrm{r}, \mathrm{s})-$ forms $\widehat{\delta}_{\tilde{g}}=(-1)^{r+s} *_{\tilde{g}}^{-1} \circ \widehat{\delta} \circ *_{\tilde{g}}$.

The supersymmetric variant of the Killing form of the s-group $A F_{n, k}^{m, l}(\Lambda)$ is degenerate. In order to generate a metric structure $\widetilde{g}_{A}$ in the total spaces of the s-bundle $\mathcal{A} N(\widetilde{E})$ we use and auxiliary nondegenerate bilinear s-form which gives rise to the possibility to define the Hodge operator $*_{\sigma_{A}}$ and $\widehat{\delta}_{\tilde{g} A}$. Applying the operator of horizontal projection $\widehat{H}$ one defines the operator $\triangle \doteq \widehat{H} \circ \widehat{\delta}_{\tilde{g} A}$ which does not depend on components of auxiliary biliniar s-form in the fiber.

Following an abstract geometric calculus, by using operators $*_{\tilde{g}}, *_{\tilde{g}_{A}}, \widehat{\delta}_{\widetilde{g}}, \widehat{\delta}_{\tilde{g} A}$ and $\triangle$ one computers

$$
\triangle \overline{\mathcal{B}}=(\triangle \mathcal{B}, \mathcal{R} t+\mathcal{R} i),
$$


where the one $\mathrm{s}-$ forms

$$
\begin{aligned}
& \mathcal{R} t=\widehat{\delta}_{\tilde{g}} \mathcal{T}+{ }_{\tilde{g}}^{-1}\left[\mathcal{F}, *_{g}^{\sim} \mathcal{T}\right\} \\
& \mathcal{R} i={ }_{\tilde{g}}^{-1}\left[\chi, *_{g}^{\sim} \mathcal{B}\right\}=(-1)^{n+k+l+m} R_{\alpha \beta} g^{\alpha \widehat{\beta}} e_{\widehat{\beta}} \delta u^{\beta}
\end{aligned}
$$

are constructed respectively by using the ds-torsions (6) and Ricci ds-tensors (8).

Let us introduce the locally anisotropic supersymmetric matter source $\overline{\mathcal{J}}$ constructed by using the same formulas from (12) when instead of $R_{\alpha \beta}$ is taken $k_{1}\left(\Upsilon_{\alpha \beta}-\frac{1}{2} g_{\alpha \beta} \Upsilon\right)-\lambda\left(g_{\alpha \beta}-\frac{1}{2} g_{\alpha \beta} \delta_{\tau}^{\tau}\right)$. By straightforward calculations we can proof $[3,4]$ that the Yang-Mills equations

$$
\triangle \overline{\mathcal{B}}=\overline{\mathcal{J}}
$$

for d-connection $\overline{\mathcal{F}}=(\mathcal{F}, \chi)$ in s-bundle $\mathcal{A} N(\widetilde{E})$, projected on the base smanifold, are equivalent to the Einstein equations (9) on $\widetilde{E}$. We emphasize that the equations (13) were introduced in a "pure" geometric manner by using operators $*, \widehat{\delta}$ and the horizontal projection $\widehat{H}$ but such gauge s-field equations are not variational because of degeneration of the Killing $\mathrm{s}-$ form. To construct a variational gauge like supersymmetric la-supergravitational model is possible, for instance, by considering a minimal extension of the gauge sgroup $A F_{n, k}^{m, l}(\Lambda)$ to the de Sitter s-group $S_{n, k}^{m, l}(\Lambda)=S O_{n, k}^{m, l}(\Lambda)$, acting on the s-space $\Lambda_{n, k}^{m, l} \oplus \Lambda$ and formulating a nonlinear version of de Sitter gauge s-gravity.

There are analyzed models of supergravity with generic local anisotropy [13] when instead of $\mathrm{s}$-field equations and constraints (9) there are considered an anholonomic generalization of the Wess-Zumino supergravity and some variants induced in low energy limit from superstring theory. The $\mathrm{N}$-connection $\mathrm{s}-$ field allows us to model generic la-interactions with dynamics and constraints induced by nontrivial (not only via toroidal compactifications) from higher dimensions and this results in a geometrical unification of the so-called generalized Finsler-Kaluza-Klein theories.

\section{$3 \quad *$-Products and Enveloping Algebras in Noncommutative Spaces}

For a noncommutative space the coordinates $\hat{u}^{i},(i=1, \ldots, N)$ satisfy some noncommutative relations of type

$$
\left[\hat{u}^{i}, \hat{u}^{j}\right]=\left\{\begin{array}{cl}
i \theta^{i j}, & \theta^{i j} \in \mathrm{IC}, \text { canonical structure; } \\
i f_{k}^{i j} \hat{u}^{k}, & f_{k}^{i j} \in \mathrm{IC}, \text { Lie structure; } \\
i C_{k l}^{i j} \hat{u}^{k} \hat{u}^{l}, & C_{k l}^{i j} \in \mathrm{IC}, \text { quantum plane structure }
\end{array}\right.
$$

where IC denotes the complex number field. 
The noncommutative space is modeled as the associative algebra of IC; this algebra is freely generated by the coordinates modulo ideal $\mathcal{R}$ generated by the relations (one accepts formal power series) $\mathcal{A}_{u}=\mathrm{IC}\left[\left[\hat{u}^{1}, \ldots, \hat{u}^{N}\right]\right] / \mathcal{R}$. One restricts attention [6] to algebras having the (so-called, Poincare-BirkhoffWitt) property that any element of $\mathcal{A}_{u}$ is defined by its coefficient function and vice versa,

$$
\widehat{f}=\sum_{L=0}^{\infty} f_{i_{1}, \ldots, i_{L}}: \hat{u}^{i_{1}} \ldots \hat{u}^{i_{L}}: \quad \text { when } \widehat{f} \sim\left\{f_{i}\right\}
$$

where $: \hat{u}^{i_{1}} \ldots \hat{u}^{i_{L}}$ : denotes that the basis elements satisfy some prescribed order (for instance, the normal order $i_{1} \leq i_{2} \leq \ldots \leq i_{L}$, or, another example, are totally symmetric). The algebraic properties are all encoded in the socalled diamond $(\diamond)$ product which is defined by

$$
\widehat{f} \widehat{g}=\widehat{h} \sim\left\{f_{i}\right\} \diamond\left\{g_{i}\right\}=\left\{h_{i}\right\} .
$$

In the mentioned approach to every function $f(u)=f\left(u^{1}, \ldots, u^{N}\right)$ of commuting variables $u^{1}, \ldots, u^{N}$ one associates an element of algebra $\widehat{f}$ when the commuting variables are substituted by anticommuting ones,

$$
f(u)=\sum f_{i_{1} \ldots i_{L}} u^{1} \cdots u^{N} \rightarrow \widehat{f}=\sum_{L=0}^{\infty} f_{i_{1}, \ldots, i_{L}}: \hat{u}^{i_{1}} \ldots \hat{u}^{i_{L}}:
$$

when the $\diamond$-product leads to a bilinear $*$-product of functions (see details in [7)

$$
\left\{f_{i}\right\} \diamond\left\{g_{i}\right\}=\left\{h_{i}\right\} \sim(f * g)(u)=h(u) .
$$

The $*$-product is defined respectively for the cases (14)

$$
f * g=\left\{\begin{array}{rc}
\left.\exp \left[\frac{i}{2} \frac{\partial}{\partial u^{i}} \theta^{i j} \frac{\partial}{\partial u^{\prime j}}\right] f(u) g\left(u^{\prime}\right)\right|_{u^{\prime} \rightarrow u}, & \text { canonical structure } \\
\left.\exp \left[\frac{i}{2} u^{k} g_{k}\left(i \frac{\partial}{\partial u^{\prime}}, i \frac{\partial}{\partial u^{\prime \prime}}\right)\right] f\left(u^{\prime}\right) g\left(u^{\prime \prime}\right)\right|_{u^{\prime \prime} \rightarrow u} ^{u^{\prime} \rightarrow u}, & \text { Lie structure; } \\
\left.q^{\frac{1}{2}\left(-u^{\prime} \frac{\partial}{\partial u^{\prime}} v \frac{\partial}{\partial v}+u \frac{\partial}{\partial u} v^{\prime} \frac{\partial}{\partial v^{\prime}}\right)} f(u, v) g\left(u^{\prime}, v^{\prime}\right)\right|_{v^{\prime} \rightarrow u} ^{u^{\prime} \rightarrow u}, & \text { quantum plane }
\end{array}\right.
$$

where there are considered values of type

$$
\begin{aligned}
e^{i k_{n} \widehat{u}^{n}} e^{i p_{n l} \widehat{u}^{n}} & =e^{i\left\{k_{n}+p_{n}+\frac{1}{2} g_{n}(k, p)\right\} \widehat{u}^{n}} \\
g_{n}(k, p) & =-k_{i} p_{j} f_{n}^{i j}+\frac{1}{6} k_{i} p_{j}\left(p_{k}-k_{k}\right) f_{m}^{i j} f_{n}^{m k}+\ldots \\
e^{A} e^{B} & =e^{A+B+\frac{1}{2}[A, B]+\frac{1}{12}([A,[A, B]]+[B,[B, A]])}+\ldots
\end{aligned}
$$

and for the coordinates on quantum (Manin) planes one holds the relation $u v=q v u$.

A non-abelian gauge theory on a noncommutative space is given by two algebraic structures, the algebra $\mathcal{A}_{u}$ and a non-abelian Lie algebra $\mathcal{A}_{I}$ of the gauge group with generators $I^{1}, \ldots, I^{S}$ and the relations

$$
\left[I^{\underline{s}}, I \underline{p}\right]=i f \frac{s \underline{p}}{\underline{t}} I^{\underline{t}} .
$$


In this case both algebras are treated on the same footing and one denotes the generating elements of the big algebra by $\widehat{u}^{i}$,

$$
\begin{aligned}
\widehat{z}^{i} & =\left\{\widehat{u}^{1}, \ldots, \widehat{u}^{N}, I^{1}, \ldots, I^{S}\right\}, \\
\mathcal{A}_{z} & =\mathrm{IC}\left[\left[\widehat{u}^{1}, \ldots, \widehat{u}^{N+S}\right]\right] / \mathcal{R},
\end{aligned}
$$

and the $*$-product formalism is to be applied for the whole algebra $\mathcal{A}_{z}$ when there are considered functions of the commuting variables $u^{i}(i, j, k, \ldots=$ $1, \ldots, N)$ and $I^{\underline{s}}(s, p, \ldots=1, \ldots, S)$.

For instance, in the case of a canonical structure for the space variables $u^{i}$ we have

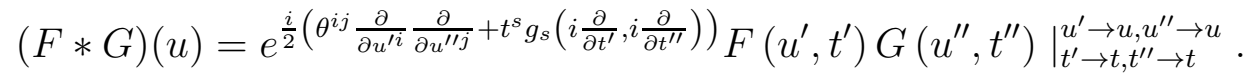

This formalism was developed in [6] for general Lie algebras. In this paper we shall consider those cases when in the commuting limit one obtains the gauge gravity and general relativity theories.

\section{Enveloping Algebras for Gravitational Gauge Connections}

To define gauge gravity theories on noncommutative space we first introduce gauge fields as elements the algebra $\mathcal{A}_{u}$ that form representation of the generator $I$-algebra for the de Sitter gauge group. For commutative spaces it is known [9, 11, 16] that an equivalent reexpression of the Einstein theory as a gauge like theory implies, for both locally isotropic and anisotropic spacetimes, the nonsemisimplicity of the gauge group, which leads to a nonvariational theory in the total space of the bundle of locally adapted affine frames (to this class one belong the gauge Poincare theories; on metric-affine and gauge gravity models see original results and reviews in [12]). By using auxililiary biliniear forms, instead of degenerated Killing form for the affine structural group, on fiber spaces, the gauge models of gravity can be formulated to be variational. After projection on the base spacetime, for the so-called Cartan connection form, the Yang-Mills equations transforms equivalently into the Einstein equations for general relativity [9]. A variational gauge gravitational theory can be also formulated by using a minimal extension of the affine structural group $\mathcal{A} f_{3+1}(\mathbb{R})$ to the de Sitter gauge group $S_{10}=S O(4+1)$ acting on $\mathbb{R}^{4+1}$ space. For cimplicity, in this paper we restrict our consideration only with the even components of frames, connections and curvatures of gauge la-supergavity outlined in previous section.

Let now consider a noncommutative space. In this case the gauge fields are elements of the algebra $\widehat{\psi} \in \mathcal{A}_{I}^{(d S)}$ that form the nonlinear representation of the de Sitter Lie algebra $s o_{(\eta)}(5)$ when the whole algebra is denoted $\mathcal{A}_{z}^{(d S)}$. Under a nonlinear de Sitter transformation the elements transform as follows

$$
\delta \widehat{\psi}=i \widehat{\gamma} \widehat{\psi}, \widehat{\psi} \in \mathcal{A}_{u}, \widehat{\gamma} \in \mathcal{A}_{z}^{(d S)} .
$$


So, the action of the generators on $\widehat{\psi}$ is defined as this element is supposed to form a nonlinear representation of $\mathcal{A}_{I}^{(d S)}$ and, in consequence, $\delta \widehat{\psi} \in \mathcal{A}_{u}$ despite $\widehat{\gamma} \in \mathcal{A}_{z}^{(d S)}$. It should be emphasized that independent of a representation the object $\widehat{\gamma}$ takes values in enveloping de Sitter algebra and not in a Lie algebra as would be for commuting spaces. The same holds for the connections that we introduce (similarly to [7]) in order to define covariant coordinates

$$
\widehat{U}^{\nu}=\widehat{u}^{v}+\widehat{\Gamma}^{\nu}, \widehat{\Gamma}^{\nu} \in \mathcal{A}_{z}^{(d S)} .
$$

The values $\widehat{U}^{\nu} \widehat{\psi}$ transforms covariantly, $\delta \widehat{U}^{\nu} \widehat{\psi}=i \widehat{\gamma} \widehat{U}^{\nu} \widehat{\psi}$, if and only if the connection $\widehat{\Gamma}^{\nu}$ satisfies the transformation law of the enveloping nonlinear realized de Sitter algerba,

$$
\delta \widehat{\Gamma}^{\nu} \widehat{\psi}=-i\left[\widehat{u}^{v}, \widehat{\gamma}\right]+i\left[\widehat{\gamma}, \widehat{\Gamma}^{\nu}\right]
$$

where $\delta \widehat{\Gamma}^{\nu} \in \mathcal{A}_{z}^{(d S)}$. The enveloping algebra-valued connection has infinitely many component fields. Nevertheless, it was shown that all the component fields can be induced from a Lie algebra-valued connection by a SeibergWitten map ([10, 5, 6] and [1] for $S O(n)$ and $S p(n))$. In this subsection we show that similar constructions could be proposed for nonlinear realizations of de Sitter algebra when the transformation of the connection is considered

$$
\delta \widehat{\Gamma}^{\nu}=-i\left[u^{\nu},{ }^{*} \widehat{\gamma}\right]+i\left[\widehat{\gamma}^{*} \widehat{\Gamma}^{\nu}\right] .
$$

For simplicity, we treat in more detail the canonical case with the star product (17). The first term in the variation $\delta \widehat{\Gamma}^{\nu}$ gives

$$
-i\left[u^{\nu},{ }^{*} \widehat{\gamma}\right]=\theta^{\nu \mu} \frac{\partial}{\partial u^{\mu}} \gamma
$$

Assuming that the variation of $\widehat{\Gamma}^{\nu}=\theta^{\nu \mu} Q_{\mu}$ starts with a linear term in $\theta$ we have

$$
\delta \widehat{\Gamma}^{\nu}=\theta^{\nu \mu} \delta Q_{\mu}, \delta Q_{\mu}=\frac{\partial}{\partial u^{\mu}} \gamma+i\left[\widehat{\gamma}^{*} Q_{\mu}\right]
$$

We follow the method of calculation from the papers [7, 6] and expand the star product (17) in $\theta$ but not in $g_{a}$ and find to first order in $\theta$,

$$
\gamma=\gamma_{\underline{a}}^{1} I^{\underline{a}}+\gamma_{\underline{a b}}^{1} I^{\underline{a}} I^{\underline{b}}+\ldots, \text { and } Q_{\mu}=q_{\mu, \underline{a}}^{1} I^{\underline{a}}+q_{\mu, \underline{a b}}^{2} I^{\underline{a}} I^{\underline{b}}+\ldots
$$

where $\gamma_{\underline{a}}^{1}$ and $q_{\mu, \underline{a}}^{1}$ are of order zero in $\theta$ and $\gamma_{\underline{a b}}^{1}$ and $q_{\mu, \underline{a b}}^{2}$ are of second order in $\theta$. The expansion in $I^{\underline{b}}$ leads to an expansion in $g_{a}$ of the $*$-product because the higher order $I^{\underline{b}}$-derivatives vanish. For de Sitter case as $I^{\underline{b}}$ we take the generators, see commutators (16), with the corresponding de Sitter structure constants $f \frac{b c}{\underline{d}} \simeq f^{\underline{\alpha}} \underline{\underline{\beta}}$ (in our further identifications with spacetime objects like frames and connections we shall use Greeck indices).

The result of calculation of variations of (18), by using $g_{a}$ to the order given in $(15)$, is

$$
\begin{aligned}
\delta q_{\mu, \underline{a}}^{1} & =\frac{\partial \gamma_{\underline{a}}^{1}}{\partial u^{\mu}}-f \frac{b c}{\underline{a}} \gamma_{\underline{b}}^{1} q_{\mu, \underline{c}}^{1}, \\
\delta Q_{\tau} & =\theta^{\mu \nu} \partial_{\mu} \gamma_{\underline{a}}^{1} \partial_{\nu} q_{\tau, \underline{I}}^{1} I^{a} I^{\underline{b}}+\ldots, \\
\delta q_{\mu, \underline{a b}}^{2} & =\partial_{\mu} \gamma_{\underline{a} \underline{b}}^{2}-\theta^{\nu \tau} \partial_{\nu} \gamma_{\underline{a}}^{1} \partial_{\tau} q_{\mu, \underline{b}}^{1}-2 f \frac{b c}{\underline{a}}\left\{\gamma_{\underline{b}}^{1} q_{\mu, \underline{c d}}^{2}+\gamma_{\underline{b d}}^{2} q_{\mu, \underline{c}}^{1}\right\} .
\end{aligned}
$$


Next we introduce the objects $\varepsilon$, taking the values in de Sitter Lie algebra and $W_{\mu}$, being enveloping de Sitter algebra valued,

$$
\varepsilon=\gamma_{\underline{a}}^{1} I^{\underline{a}} \text { and } W_{\mu}=q_{\mu, \underline{a b}}^{2} I^{\underline{a}} I^{\underline{b}}
$$

with the variation $\delta W_{\mu}$ satisfying the equation [7, 6]

$$
\delta W_{\mu}=\partial_{\mu}\left(\gamma_{\underline{a b}}^{2} I^{\underline{a}} I^{\underline{b}}\right)-\frac{1}{2} \theta^{\tau \lambda}\left\{\partial_{\tau} \varepsilon, \partial_{\lambda} q_{\mu}\right\}+i\left[\varepsilon, W_{\mu}\right]+i\left[\left(\gamma_{\underline{a b}}^{2} I^{\underline{a}} I^{\underline{b}}\right), q_{\nu}\right] .
$$

The equation (19) has the solution (found in [7, 10])

$$
\gamma_{\underline{a b}}^{2}=\frac{1}{2} \theta^{\nu \mu}\left(\partial_{\nu} \gamma_{\underline{a}}^{1}\right) q_{\mu, \underline{b}}^{1}, \text { and } q_{\mu, \underline{a b}}^{2}=-\frac{1}{2} \theta^{\nu \tau} q_{\nu, \underline{a}}^{1}\left(\partial_{\tau} q_{\mu, \underline{b}}^{1}+R_{\tau \mu, \underline{b}}^{1}\right)
$$

where $R_{\tau \mu, \underline{b}}^{1}=\partial_{\tau} q_{\mu, \underline{b}}^{1}-\partial_{\mu} q_{\tau, \underline{b}}^{1}+f \frac{e c}{\underline{d}} q_{\tau, \underline{e}}^{1} q_{\mu, \underline{e}}^{1}$ can be identified with the coefficients $\mathcal{R}^{\underline{\alpha}}{ }_{\underline{\beta} \mu \nu}$ of de Sitter nonlinear gauge gravity curvature if in the commutative limit $q_{\mu, \underline{b}}^{1} \simeq\left(\begin{array}{cc}\Gamma^{\underline{\alpha}} & l_{0}^{-1} \chi^{\underline{\alpha}} \\ l_{0}^{-1} \frac{\chi_{\underline{\beta}}}{2} & 0\end{array}\right)$.

The presented procedure can be generalized to all higher powers of $\theta[6]$.

\section{Noncommutative Gauge Gravity Covariant Dynamics}

The constructions from the previous section are summarized by the conclusion that the de Sitter algebra valued object $\varepsilon=\gamma_{\underline{a}}^{1}(u) I^{\underline{a}}$ determines all the terms in the enveloping algebra

$$
\gamma=\gamma_{\underline{a}}^{1} I^{\underline{a}}+\frac{1}{4} \theta^{\nu \mu} \partial_{\nu} \gamma_{\underline{a}}^{1} q_{\mu, \underline{b}}^{1}\left(I^{\underline{a}} I^{\underline{b}}+I^{\underline{b}} I^{\underline{a}}\right)+\ldots
$$

and the gauge transformations are defined by $\gamma_{\underline{a}}^{1}(u)$ and $q_{\mu, \underline{b}}^{1}(u)$, when

$$
\delta_{\gamma^{1}} \psi=i \gamma\left(\gamma^{1}, q_{\mu}^{1}\right) * \psi
$$

For de Sitter enveloping algebras one holds the general formula for compositions of two transformations

$$
\delta_{\gamma} \delta_{\varsigma}-\delta_{\varsigma} \delta_{\gamma}=\delta_{i(\varsigma * \gamma-\gamma * \varsigma)}
$$

which holds also for the restricted transformations defined by $\gamma^{1}$,

$$
\delta_{\gamma^{1}} \delta_{\varsigma^{1}}-\delta_{\varsigma^{1}} \delta_{\gamma^{1}}=\delta_{i\left(\varsigma^{1} * \gamma^{1}-\gamma^{1} * \varsigma^{1}\right)} .
$$

Applying the formula (17) we computer

$$
\begin{aligned}
{\left[\gamma,{ }^{*} \zeta\right]=} & i \gamma_{\underline{a}}^{1} \zeta_{\underline{b}}^{1} f \frac{a b}{\underline{c}} I^{\underline{c}}+\frac{i}{2} \theta^{\nu \mu}\left\{\partial_{v}\left(\gamma_{\underline{a}}^{1} \zeta_{\underline{b}}^{1} f \frac{a b}{\underline{c}}\right) q_{\mu, \underline{c}}\right. \\
& \left.+\left(\gamma_{\underline{a}}^{1} \partial_{v} \zeta_{\underline{b}}^{1}-\zeta_{\underline{a}}^{1} \partial_{v} \gamma_{\underline{b}}^{1}\right) q_{\mu, \underline{b}} f \frac{a b}{\underline{a}}+2 \partial_{v} \gamma_{\underline{a}}^{1} \partial_{\mu} \zeta_{\underline{b}}^{1}\right\} I^{\underline{d}} I^{\underline{c}} .
\end{aligned}
$$


Such commutators could be used for definition of tensors [7]

$$
\widehat{S}^{\mu \nu}=\left[\widehat{U}^{\mu}, \widehat{U}^{\nu}\right]-i \widehat{\theta}^{\mu \nu}
$$

where $\hat{\theta}^{\mu \nu}$ is respectively stated for the canonical, Lie and quantum plane structures. Under the general enveloping algebra one holds the transform

$$
\delta \widehat{S}^{\mu \nu}=i\left[\widehat{\gamma}, \widehat{S}^{\mu \nu}\right]
$$

For instance, the canonical case is characterized by

$$
\begin{aligned}
S^{\mu \nu} & =i \theta^{\mu \tau} \partial_{\tau} \Gamma^{\nu}-i \theta^{\nu \tau} \partial_{\tau} \Gamma^{\mu}+\Gamma^{\mu} * \Gamma^{\nu}-\Gamma^{\nu} * \Gamma^{\mu} \\
& =\theta^{\mu \tau} \theta^{\nu \lambda}\left\{\partial_{\tau} Q_{\lambda}-\partial_{\lambda} Q_{\tau}+Q_{\tau} * Q_{\lambda}-Q_{\lambda} * Q_{\tau}\right\}
\end{aligned}
$$

By introducing the gravitational gauge strength (curvature)

$$
R_{\tau \lambda}=\partial_{\tau} Q_{\lambda}-\partial_{\lambda} Q_{\tau}+Q_{\tau} * Q_{\lambda}-Q_{\lambda} * Q_{\tau}
$$

which could be treated as a noncommutative extension of de Sitter nonlinear gauge gravitational curvature one computers

$$
R_{\tau \lambda, \underline{a}}=R_{\tau \lambda, \underline{a}}^{1}+\theta^{\mu \nu}\left\{R_{\tau \mu, \underline{a}}^{1} R_{\lambda \nu, \underline{b}}^{1}-\frac{1}{2} q_{\mu, \underline{a}}^{1}\left[\left(D_{\nu} R_{\tau \lambda, \underline{b}}^{1}\right)+\partial_{\nu} R_{\tau \lambda, \underline{b}}^{1}\right]\right\} I^{\underline{b}},
$$

where the gauge gravitation covariant derivative is introduced,

$$
\left(D_{\nu} R_{\tau \lambda, \underline{b}}^{1}\right)=\partial_{\nu} R_{\tau \lambda, \underline{b}}^{1}+q_{\nu, \underline{c}} R_{\tau \lambda, \underline{d}}^{1} f \frac{c d}{\underline{b}} .
$$

Following the gauge transformation laws for $\gamma$ and $q^{1}$ we find

$$
\delta_{\gamma^{1}} R_{\tau \lambda}^{1}=i\left[\gamma,{ }^{*} R_{\tau \lambda}^{1}\right]
$$

with the restricted form of $\gamma$.

Such formulas were proved in references [6, 10] for usual gauge (nongravitational) fields. Here we reconsidered them for gravitational gauge fields.

Following the nonlinear realization of de Sitter algebra and the $*$-formalism we can formulate a dynamics of noncommutative spaces. Derivatives can be introduced in such a way that one does not obtain new relations for the coordinates. In this case a Leibniz rule can be defined [6] that

$$
\widehat{\partial}_{\mu} \widehat{u}^{\nu}=\delta_{\mu}^{\nu}+d_{\mu \sigma}^{\nu \tau} \widehat{u}^{\sigma} \widehat{\partial}_{\tau}
$$

where the coefficients $d_{\mu \sigma}^{\nu \tau}=\delta_{\sigma}^{\nu} \delta_{\mu}^{\tau}$ are chosen to have not new relations when $\widehat{\partial}_{\mu}$ acts again to the right hand side. In consequence one holds the $*$-derivative formulas

$$
\begin{gathered}
\partial_{\tau} * f=\frac{\partial}{\partial u^{\tau}} f+f * \partial_{\tau}, \\
{\left[\partial_{l}{ }^{*}(f * g)\right]=\left(\left[\partial_{l},{ }^{*} f\right]\right) * g+f *\left(\left[\partial_{l},{ }^{*} g\right]\right)}
\end{gathered}
$$


and the Stokes theorem $\int\left[\partial_{l}, f\right]=\int d^{N} u\left[\partial_{l},{ }^{*} f\right]=\int d^{N} u \frac{\partial}{\partial u^{l}} f=0$, where, for the canonical structure, the integral is defined,

$$
\int \widehat{f}=\int d^{N} u f\left(u^{1}, \ldots, u^{N}\right) .
$$

An action can be introduced by using such integrals. For instance, for a tensor of type (20), when $\delta \widehat{L}=i[\widehat{\gamma}, \widehat{L}]$, we can define a gauge invariant action

$$
W=\int d^{N} u \operatorname{Tr} \widehat{L}, \delta W=0
$$

were the trace has to be taken for the group generators.

For the nonlinear de Sitter gauge gravity a proper action is

$$
L=\frac{1}{4} R_{\tau \lambda} R^{\tau \lambda}
$$

where $R_{\tau \lambda}$ is defined by the even part of (11). In this case the dynamic of noncommutative space is entirely formulated in the framework of quantum field theory of gauge fields. The method works for matter fields as well to restrictions to the general relativity theory (see references [11, 9]).

Acknowledgement: The first author (S. V.) is grateful to Organizes of NATO ARW in Kiev, where the results of this work were communicated, for kind hospitality and support.

\section{References}

[1] L. Bonora, M. Schnabl, M. M. Sheikh-Jabbari and A. Tomasiello, Noncommutative $S O(n)$ and $S p(n)$ gauge theories, hep-th/0006091.

[2] A. Connes, J. Math. Phys. 36 (1995) 6194; A. H. Chamseddine, A. Connes, Phys. Rev. Letters 77 (1996) 4868; L. Carminati, B. Iochum, T. Schöcer, Noncommutative Yang-Mills and noncommutative relativity: a bridge over troubled water, hep-th/9706105.

[3] A. Connes, M. R. Douglas and A. Schwarz, JHEP 9802 (1998) 003, hep th/0001203.

[4] E. Hawkins, Comm. Math. Phys. 187 (1997) 471; gr-qc / 9605068; G. Landi, N. A. Viet, K. C. Wali, Phys. Lett. B326 (1994) 45; hep-th / 9402046; A, Sitarz, Class. Quant. Grav. 11 (1994) 2127; hep-th / 9401145; J. Madore and J. Mourad, Int. J. Mod. Phys. D3 (1994) 221.

[5] B. Jurčo and P. Schupp, Eur. Phys. J. C 14, (2000) 367, hep-th/0001032.

[6] B. Jurčo, S. Schraml, P. Shupp and J. Wess, Enveloping algebra valued gauge transformations for non-abelian gauge groups on non-commutative spaces, hep-th/0006246 
[7] J. Madore, S. Schraml, P. Schupp and J. Wess, Gauge theory on noncommutative spaces, Eur. Phys. J. C, in press, hep-th/0001203.

[8] S. Majid, Conceptual Issues for Noncommutative gravity and algebras and finite sets, math.QA / 0006152; A. H. Chamseddine, Complexified gravity and noncommutative spaces, hep-th / 0005222; R. Kerner, Noncommutative extensions of classical theories in physics, hep-th / 0004033.

[9] D. A. Popov, Theor. Math. Phys. 24 (1975) 347; D. A. Popov and L. I. Dikhin, Doklady Akademii Nauk SSSR 245 (1975) 347 [in Russian].

[10] N. Seiberg and E. Witten, JHEP 9909 (1999) 032, hep-th/9908142.

[11] A. A. Tseytlin, Phys. Rev. D 26 (1982) 3327.

[12] R. Utiyama, Phys. Rev. 101 (1956) 1597; V. N. Ponomariov, A. Barvinsky and Yu. N. Obukhov, Geometrodynamical Methods and the Gauge Approach to the Gravitational Interactions (Energoatomizdat, Moscow, 1985); E. W. Mielke, Geometrodynamics of Gauge Fields - on the Geometry of Yang-Mills and Gravitational Gauge Theories (Academic-Verlag, Berlin, 1987); F. Hehl, J. D. McGrea, E. W. Mielke and Y. Ne'eman, Phys. Rep. 258 (1995) 1; H. Dehnen and E. Hitzer, Int. J. Theor. Phys. 34 (1995) 1981.

[13] S. Vacaru, Nucl. Phys. B 434 (1997) 590; gr-qc/9604016; hep-th / 9604194-9604196; Interactions, Strings and Isotopies in Higher Order Anisotropic Superspaces (Hadronic Press, 1998).

[14] S. Vacaru, Gauge and Eistein gravity from non-Abelian gauge models on noncommutative spaces, hep-th/0009163

[15] S. Vacaru and H. Dehnen, Locally Anisotropic Structures and Nonlinear Connections in Einstein and Gauge Gravity, gr-qc / 0009039.

[16] S. Vacaru and Yu. Goncharenko, Int. J. Theor. Phys. 34 (1995) 1955.

[17] J. Wess and B. Zumino, Nucl. Phys. Proc. Suppl. 18B (1991) 302; J. Wess, in Proceeding of the 38 International Universitätswochen für Kern- und Teilchenphysik, no. 543 in Lect. Notes in Phys., Springer-Verlag, 2000, Schladming, January 1999, eds. H. Gusterer, H. Grosse and L. Pitner; math-ph / 9910013.

[18] H. Weyl, Z. Physik 46 (1927) 1; The theory of groups and quantum mechanics (Dover, New-York, 1931), translated from Gruppentheorie and Quantenmechanik (Hirzel Verlag, Leipzig, 1928); E. P. Wigner, Phys. Rev. 40 (1932) 749; J. E. Moyal, Proc. Cambridge Phil. Soc. 45 (1949) 99; F. Bayen, M. Flato, C. Fronsdal, A. Lichnerowicz, D. Sternheimer, Ann. Physics 111 (1978) 61; M. Kontsevitch, Deformation quantization of Poisson manifolds, I. q-alg/9709040; D. Sternheimer, Deformation Quantization: Twenty Years After, math / 9809056. 